\title{
Application of microwave-induced combustion and isotope dilution strategies for quantification of sulfur in coals via sector-field induc- tively coupled plasma mass spectrometry
}

\section{Supplemental Information}

\author{
Corresponding Author \\ * Steven J. Christopher \\ NIST Material Measurement Laboratory \\ Chemical Sciences Division \\ Environmental Chemical Sciences Group \\ NIST Charleston Laboratory \\ 331 Fort Johnson Road \\ Charleston, SC 29412
}

\author{
Co-Author \\ tThomas W. Vetter \\ NIST Material Measurement Laboratory \\ Chemical Sciences Division \\ Inorganic Measurement Science Group \\ MS 8391 \\ Gaithersburg, MD 20899
}

Table of Contents

Supplemental Table 1. Quantity descriptions for each variable in the sulfur isotope dilution measurement function

Supplemental Table 2. Example input table for SRM 2682c showing the calculated $\mathrm{S}$ mass fraction result $c_{\mathrm{x}}$, and corresponding metadata used to determine the mean and expanded uncertainty for a single permutation of the IDMS measurement function.
Supplemental Table 3. Uncertainty descriptions and estimates for each variable in the sulfur isotope dilution measurement function.............................

Supplemental Figure 1. Absolute ID-MIC-SF-ICPMS procedure blanks in units of micrograms with associated standard uncertainties, showing reduction of average blanks as a function of method development progression..............................................

Supplemental Figure 2. Truncated Kragten spreadsheet for SRM 2682c S-7 
Supplemental Table 1. Quantity descriptions for each variable in the sulfur isotope dilution measurement function.

\begin{tabular}{|c|c|c|c|c|c|}
\hline $\begin{array}{l}\text { Quantity } \\
\text { Symbol }\end{array}$ & Quantity Description & $\begin{array}{l}\text { Quantity } \\
\text { Symbol }\end{array}$ & Quantity Description & $\begin{array}{l}\text { Quantity } \\
\text { Symbol }\end{array}$ & Quantity Description \\
\hline$c_{\mathrm{x}}(\%)$ & mass fraction of test portion & $\begin{array}{c}c_{\mathrm{blk}} \\
(\mu \mathrm{mol} / \mathrm{g})\end{array}$ & $\begin{array}{l}\text { amount content of procedure } \\
\text { blank }\end{array}$ & $\begin{array}{c}K_{\mathrm{x} 1} \\
\text { (unitless) }\end{array}$ & $\begin{array}{l}\text { mass bias correction of } \\
R_{\mathrm{x} 1}(32 / 34) \text { in sample }\end{array}$ \\
\hline $\begin{array}{c}a_{\mathrm{z}} \\
(\mathrm{g} / \mathrm{mol})\end{array}$ & $\begin{array}{l}\text { primary standard molecular } \\
\text { mass (SRM 3154) }\end{array}$ & $\begin{array}{c}K_{\mathrm{b}} \\
\text { (unitless) }\end{array}$ & $\begin{array}{l}\text { mass bias correction of } R_{b} \\
(34 / 32)\end{array}$ & $\begin{array}{c}K_{\mathrm{x} 2} \\
\text { (unitless) }\end{array}$ & $\begin{array}{l}\text { mass bias correction of } \\
R_{\mathrm{x} 2}(33 / 32) \text { in sample }\end{array}$ \\
\hline $\begin{array}{c}a_{\mathrm{x}} \\
(\mathrm{g} / \mathrm{mol})\end{array}$ & sulfur molecular mass & $\begin{array}{c}K_{b}^{\prime} \\
\text { (unitless) }\end{array}$ & $\begin{array}{l}\text { mass bias correction of } \mathrm{R}_{\mathrm{b}}^{\prime} \\
(34 / 32)\end{array}$ & $\begin{array}{c}K_{\mathrm{x} 3} \\
\text { (unitless) }\end{array}$ & $\begin{array}{l}\text { mass bias correction of } \\
R_{\mathrm{x} 3}(32 / 32) \text { in sample }\end{array}$ \\
\hline $\begin{array}{c}d_{\mathrm{x}} \\
\text { (unitless) }\end{array}$ & $\begin{array}{l}\text { test portion drying correction } \\
\text { factor }\end{array}$ & $\begin{array}{c}K_{\mathrm{y} 1} \\
\text { (unitless) }\end{array}$ & mass bias correction of $\mathrm{R}_{\mathrm{y} 1}$ & $\begin{array}{c}K_{\mathrm{x} 4} \\
\text { (unitless) }\end{array}$ & $\begin{array}{l}\text { mass bias correction of } \\
R_{\mathrm{x} 4}(36 / 32) \text { in sample }\end{array}$ \\
\hline $\begin{array}{l}m_{\mathrm{x}} \\
(\mathrm{g})\end{array}$ & mass of test portion in blend & $\begin{array}{c}R_{\mathrm{x} 1} \\
\text { (unitless) }\end{array}$ & $\begin{array}{c}\text { ratio sample-measured ratio } \\
\text { of enriched isotope to refer- } \\
\text { ence isotope }(34 / 32)\end{array}$ & $\begin{array}{c}K_{\mathrm{z} 1} \\
\text { (unitless) }\end{array}$ & $\begin{array}{c}\text { mass bias correction of } \\
R_{\mathrm{z} 1}(34 / 32) \text { in primary } \\
\text { standard }\end{array}$ \\
\hline $\begin{array}{c}m_{y}^{\prime} \\
(g)\end{array}$ & $\begin{array}{l}\text { mass of spike in spike calibra- } \\
\text { tion }\end{array}$ & $\begin{array}{c}R_{\mathrm{x} 2} \\
\text { (unitless) }\end{array}$ & ratio sample $(33 / 32)$ & $\begin{array}{c}K_{\mathrm{z2}} \\
\text { (unitless) }\end{array}$ & $\begin{array}{l}\text { mass bias correction of } \\
R_{\mathrm{z} 2}(33 / 32) \text { in primary } \\
\text { standard }\end{array}$ \\
\hline $\begin{array}{c}R_{\mathrm{b}} \\
\text { (unitless) }\end{array}$ & $\begin{array}{l}\text { measured ratio of blend } 34 / 32 \\
\text { spike isotope/reference isotope }\end{array}$ & $\begin{array}{c}R_{\mathrm{x} 3} \\
\text { (unitless) }\end{array}$ & ratio sample $(32 / 32)$ & $\begin{array}{c}K_{\mathrm{z3}} \\
\text { (unitless) }\end{array}$ & $\begin{array}{c}\text { mass bias correction of } \\
R_{\mathrm{z} 3}(32 / 32) \text { in primary } \\
\text { standard }\end{array}$ \\
\hline $\begin{array}{c}R_{b}^{\prime} \\
\text { (unitless) }\end{array}$ & $\begin{array}{c}\text { measured ratio of spike calibra- } \\
\text { tion back blend (34/32) spike } \\
\text { isotope/reference isotope }\end{array}$ & $\begin{array}{c}R_{\mathrm{x} 4} \\
\text { (unitless) }\end{array}$ & ratio sample $(36 / 32)$ & $\begin{array}{c}K_{\mathrm{z4}} \\
\text { (unitless) }\end{array}$ & $\begin{array}{c}\text { mass bias correction of } \\
R_{\mathrm{z} 4}(36 / 32) \text { in primary } \\
\text { standard }\end{array}$ \\
\hline $\begin{array}{c}R_{\mathrm{y} 1} \\
\text { (unitless) }\end{array}$ & $\begin{array}{l}\text { measured ratio of } 34 \text { spike iso- } \\
\text { tope to } 32 \text { reference isotope in } \\
\text { the }{ }^{34} \mathrm{~S} \text { enriched spike material }\end{array}$ & $\begin{array}{c}R_{\mathrm{z} 1} \\
\text { (unitless) }\end{array}$ & $\begin{array}{c}\text { ratio primary standard- } \\
\text { measured ratio of enriched } \\
\text { isotope to reference isotope } \\
(34 / 32)\end{array}$ & $\begin{array}{c}C_{\text {Rep }} \\
\text { (unitless) }\end{array}$ & $\begin{array}{l}\text { Constant }=1 \text { used to in- } \\
\text { corporate replication un- } \\
\text { certainty }\end{array}$ \\
\hline $\begin{array}{l}m_{\mathbf{y}} \\
(g)\end{array}$ & mass of enriched spike in blend & $\begin{array}{c}R_{\mathrm{z2}} \\
\text { (unitless) }\end{array}$ & $\begin{array}{l}\text { ratio primary standard } \\
\qquad(33 / 32)\end{array}$ & $\begin{array}{c}C_{\mathrm{dt}} \\
\text { (unitless) }\end{array}$ & $\begin{array}{l}\text { Constant }=1 \text { used to in- } \\
\text { corporate detector dead } \\
\text { time uncertainty }\end{array}$ \\
\hline $\begin{array}{l}m_{\mathrm{z}} \\
(\mathrm{g})\end{array}$ & $\begin{array}{l}\text { mass of primary assay standard } \\
\text { in spike calibration back blend }\end{array}$ & $\begin{array}{c}R_{\mathrm{z3}} \\
\text { (unitless) }\end{array}$ & $\begin{array}{l}\text { ratio primary standard } \\
\qquad(32 / 32)\end{array}$ & $\begin{array}{c}C_{\mathrm{bgd}} \\
\text { (unitless) }\end{array}$ & $\begin{array}{l}\text { Constant }=1 \text { used to in- } \\
\text { corporate background } \\
\text { subtraction uncertainty }\end{array}$ \\
\hline $\begin{array}{c}c_{\mathrm{z}} \\
(\mu \mathrm{mol} / \mathrm{g})\end{array}$ & $\begin{array}{c}\text { amount content of primary assay } \\
\text { standard }\end{array}$ & $\begin{array}{c}R_{\mathrm{z} 4} \\
\text { (unitless) }\end{array}$ & $\begin{array}{c}\text { ratio primary standard } \\
(36 / 32)\end{array}$ & & \\
\hline
\end{tabular}


Supplemental Table 2. Example input table for SRM 2682c showing the calculated S mass fraction result $c_{\mathrm{x}}$, and corresponding metadata used to determine the mean and expanded uncertainty for a single permutation of the ID-MIC-SF-ICPMS measurement function ( 1 of 32 possible permutations, based on individual sample and spike solution pairs). The metadata are fed into a Kragten spreadsheet to estimate the uncertainty of the ID-MIC-SF-ICPMS method. The data for each quantity are presented in the following pattern: quantity value, reported uncertainty (standard deviation), method of evaluating uncertainty (Type A or Type B), standard uncertainty and degrees of freedom. Reported uncertainties evaluated by Type A methods were normalized to standard uncertainties by division by $\sqrt{n}$. Reported uncertainties with evaluated by Type B methods were modeled as rectangular distributions (normalized to standard uncertainties by division by $\sqrt{3}$ ), with the exception of published expanded uncertainties, which were normalized to standard uncertainties using the published coverage factor. The S mass fraction data for SRM 2682c described in the text are based on the average of all permutations $(0.4902 \% \pm 0.0068 \%)$.

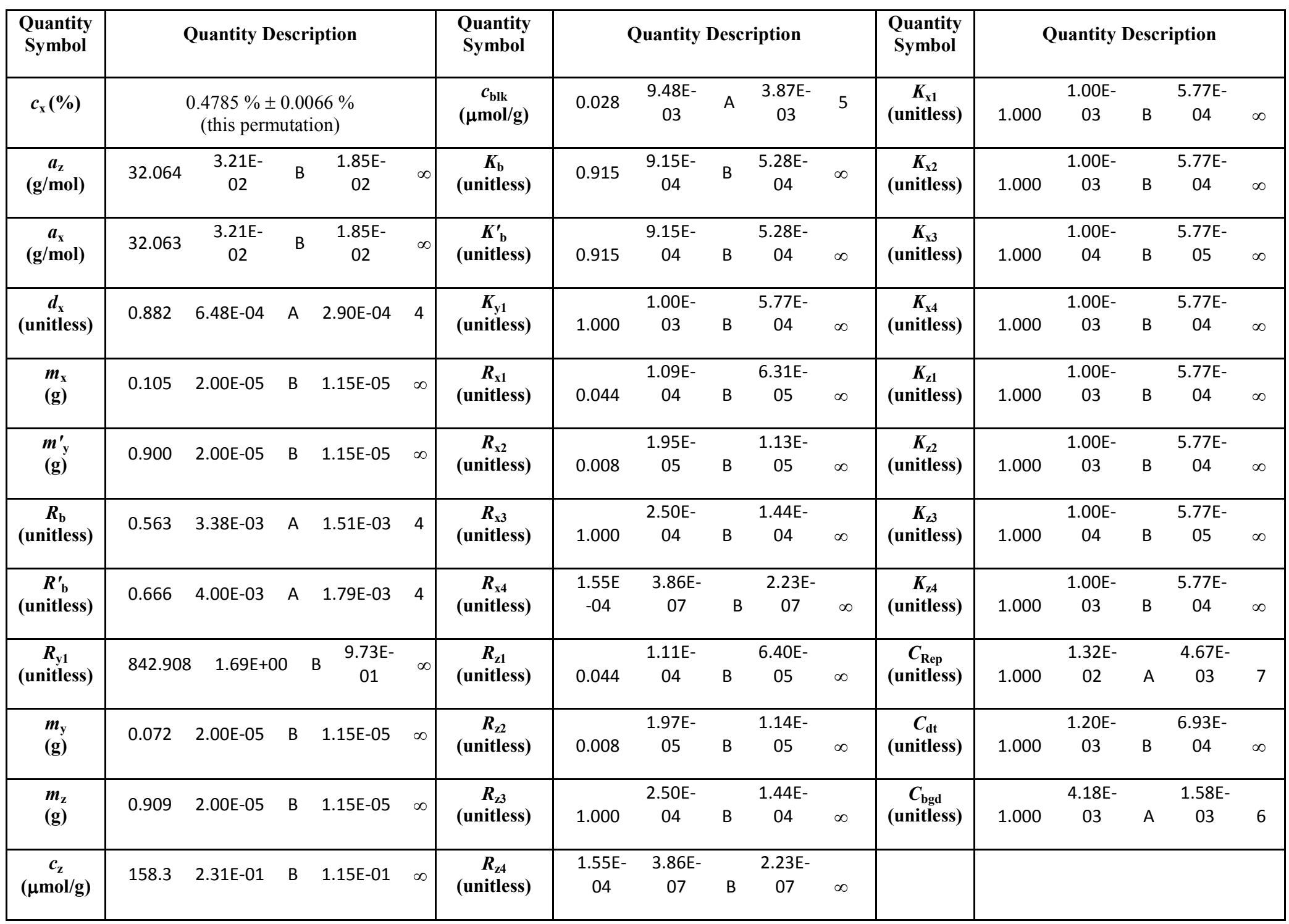


Supplemental Table 3. Uncertainty descriptions and estimates for each variable in the ID-MIC-SF-ICPMS measurement function. Reported uncertainties evaluated by Type A methods were normalized to standard uncertainties by division by $\sqrt{n}$. Reported uncertainties evaluated by Type B methods were modeled as rectangular distributions (normalized to standard uncertainties by division by $\sqrt{3}$ ), with the exception of published expanded uncertainties, which were normalized to standard uncertainties using the published coverage factor.

\begin{tabular}{|c|c|c|c|c|c|}
\hline $\begin{array}{l}\text { Quantity } \\
\text { Symbol }\end{array}$ & Uncertainty Description & $\begin{array}{l}\text { Quantity } \\
\text { Symbol }\end{array}$ & Uncertainty Description & $\begin{array}{l}\text { Quantity } \\
\text { Symbol }\end{array}$ & $\begin{array}{l}\text { Uncertainty } \\
\text { Description }\end{array}$ \\
\hline $\begin{array}{c}a_{\mathrm{z}} \\
(\mathrm{g} / \mathrm{mol})\end{array}$ & $\begin{array}{l}\text { assume } 0.1 \% \text { of quantity value, based on } \\
\text { two times the IUPAC range of possible } \mathrm{S} \\
\text { atomic weights (range }=32.059 \mathrm{~g} / \mathrm{mol} \text { to } \\
\qquad 32.076 \mathrm{~g} / \mathrm{mol})\end{array}$ & $\begin{array}{c}K_{\mathrm{b}} \\
\text { (unitless) }\end{array}$ & $\begin{array}{c}\text { assume } 0.1 \% \text { of quantity } \\
\text { value }\end{array}$ & $\begin{array}{c}K_{\mathrm{x} 2} \\
\text { (unitless) }\end{array}$ & $\begin{array}{l}\text { assume } 0.1 \% \text { of } \\
\text { quantity value }\end{array}$ \\
\hline $\begin{array}{c}a_{\mathrm{x}} \\
(\mathrm{g} / \mathrm{mol})\end{array}$ & $\begin{array}{c}\text { assume } 0.1 \% \text { of quantity value, based on } \\
\text { two times the IUPAC range of possible } \mathrm{S} \\
\text { atomic weights (range }=32.059 \mathrm{~g} / \mathrm{mol} \text { to } \\
\qquad 32.076 \mathrm{~g} / \mathrm{mol})^{1}\end{array}$ & $\begin{array}{c}K_{b}^{\prime} \\
\text { (unitless) }\end{array}$ & $\begin{array}{c}\text { assume } 0.1 \% \text { of quantity } \\
\text { value }\end{array}$ & $\begin{array}{c}K_{\mathrm{x} 3} \\
\text { (unitless) }\end{array}$ & $\begin{array}{c}\text { assume } 0.01 \% \text { of } \\
\text { quantity value }= \\
1\end{array}$ \\
\hline $\begin{array}{c}d_{\mathrm{x}} \\
\text { (unitless) }\end{array}$ & $\begin{array}{c}\text { absolute } u_{\mathrm{i}} \text { for } 5 \text { moisture factor replicates } \\
\text { from same bottle }\end{array}$ & $\begin{array}{c}K_{\mathrm{y} 1} \\
\text { (unitless) }\end{array}$ & $\begin{array}{c}\text { assume } 0.1 \% \text { of quantity } \\
\text { value }\end{array}$ & $\begin{array}{c}K_{\mathrm{x} 4} \\
\text { (unitless) }\end{array}$ & $\begin{array}{l}\text { assume } 0.1 \% \text { of } \\
\text { quantity value }\end{array}$ \\
\hline $\begin{array}{l}m_{\mathbf{x}} \\
(\mathrm{g})\end{array}$ & $\begin{array}{l}\text { two times the 5-place balance readability } \\
\text { of } 0.00001 \mathrm{~g} \text {, }\end{array}$ & $\begin{array}{c}R_{\mathrm{x} 1} \\
\text { (unitless) }\end{array}$ & $\begin{array}{l}\text { assume } 0.25 \% \text { of quantity } \\
\text { value, based on usual in- } \\
\text { strumental measurement } \\
\text { precision }\end{array}$ & $\begin{array}{c}K_{\mathrm{z} 1} \\
\text { (unitless) }\end{array}$ & $\begin{array}{l}\text { assume } 0.1 \% \text { of } \\
\text { quantity value }\end{array}$ \\
\hline $\begin{array}{l}m_{y}^{\prime} \\
(g)\end{array}$ & $\begin{array}{l}\text { two times the 5-place balance readability } \\
\text { of } 0.00001 \mathrm{~g}\end{array}$ & $\begin{array}{c}R_{\mathrm{x} 2} \\
\text { (unitless) }\end{array}$ & $\begin{array}{l}\text { assume } 0.25 \% \text { of quantity } \\
\text { value, based on usual in- } \\
\text { strumental measurement } \\
\text { precision }\end{array}$ & $\begin{array}{c}K_{\mathrm{z2}} \\
\text { (unitless) }\end{array}$ & $\begin{array}{l}\text { assume } 0.1 \% \text { of } \\
\text { quantity value }\end{array}$ \\
\hline $\begin{array}{c}R_{\mathrm{b}} \\
\text { (unitless) }\end{array}$ & $\begin{array}{l}\% \text { RSD of } 5 \text { replicate isotope ratio meas- } \\
\text { urements across experiment run }\end{array}$ & $\begin{array}{c}R_{\mathrm{x} 3} \\
\text { (unitless) }\end{array}$ & $\begin{array}{c}\text { assume } 0.025 \% \text { of quantity } \\
\text { value }=1\end{array}$ & $\begin{array}{c}K_{\mathrm{z3}} \\
\text { (unitless) }\end{array}$ & $\begin{array}{c}\text { assume } 0.01 \% \text { of } \\
\text { quantity value }= \\
1\end{array}$ \\
\hline $\begin{array}{c}R_{b}^{\prime} \\
\text { (unitless) }\end{array}$ & $\begin{array}{l}\% \text { RSD of } 5 \text { replicate isotope ratio meas- } \\
\text { urements across experiment run }\end{array}$ & $\begin{array}{c}R_{\mathrm{x} 4} \\
\text { (unitless) }\end{array}$ & $\begin{array}{l}\text { assume } 0.25 \% \text { of quantity } \\
\text { value, based on usual in- } \\
\text { strumental measurement } \\
\text { precision }\end{array}$ & $\begin{array}{c}K_{\mathrm{z4}} \\
\text { (unitless) }\end{array}$ & $\begin{array}{l}\text { assume } 0.1 \% \text { of } \\
\text { quantity value }\end{array}$ \\
\hline
\end{tabular}

1. Atomic Weights of the Elements 2011 (IUPAC Technical Report), Pure Appl. Chem. 2013, 85, 5, 1047-1078. 
Supplemental Table 3 (continued). Uncertainty descriptions and estimates for each variable in the ID-MIC-SF-ICPMS measurement function. Reported uncertainties evaluated by Type A methods were normalized to standard uncertainties by division by $\sqrt{n}$. Reported uncertainties evaluated by Type B methods were modeled as rectangular distributions (normalized to standard uncertainties by division by $\sqrt{3}$ ), with the exception of published expanded uncertainties, which were normalized to standard uncertainties using the published coverage factor.

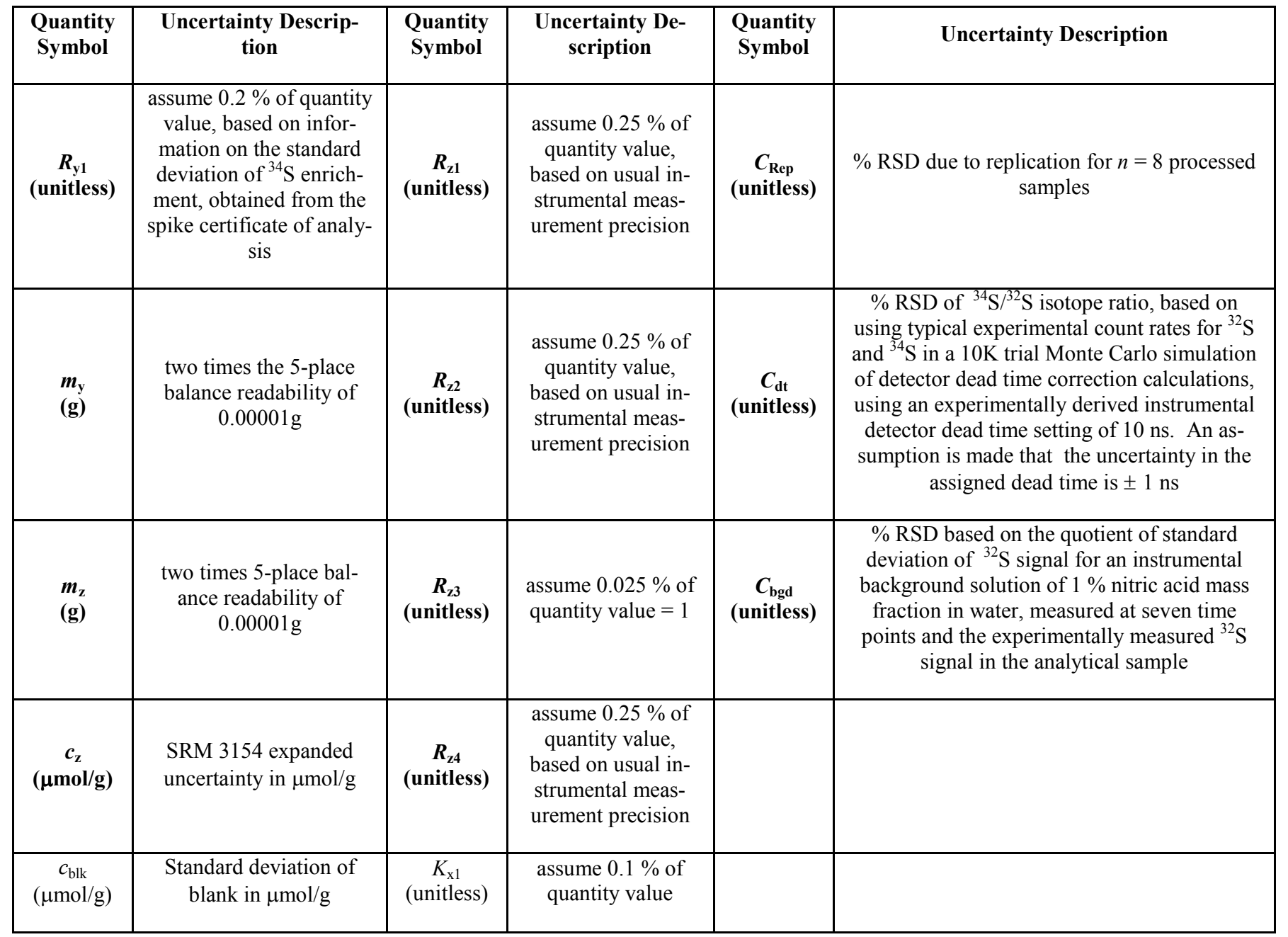


Supplemental Figure 1. Absolute ID-MIC-SF-ICPMS procedure blanks in units of micrograms with associated standard uncertainties, showing reduction of average blanks as a function of method development progression. The number of blanks were $n=5$, for the SRM $2685 \mathrm{~b}$ project and $n=6$ for both the SRM 2684b and SRM 2682c projects.

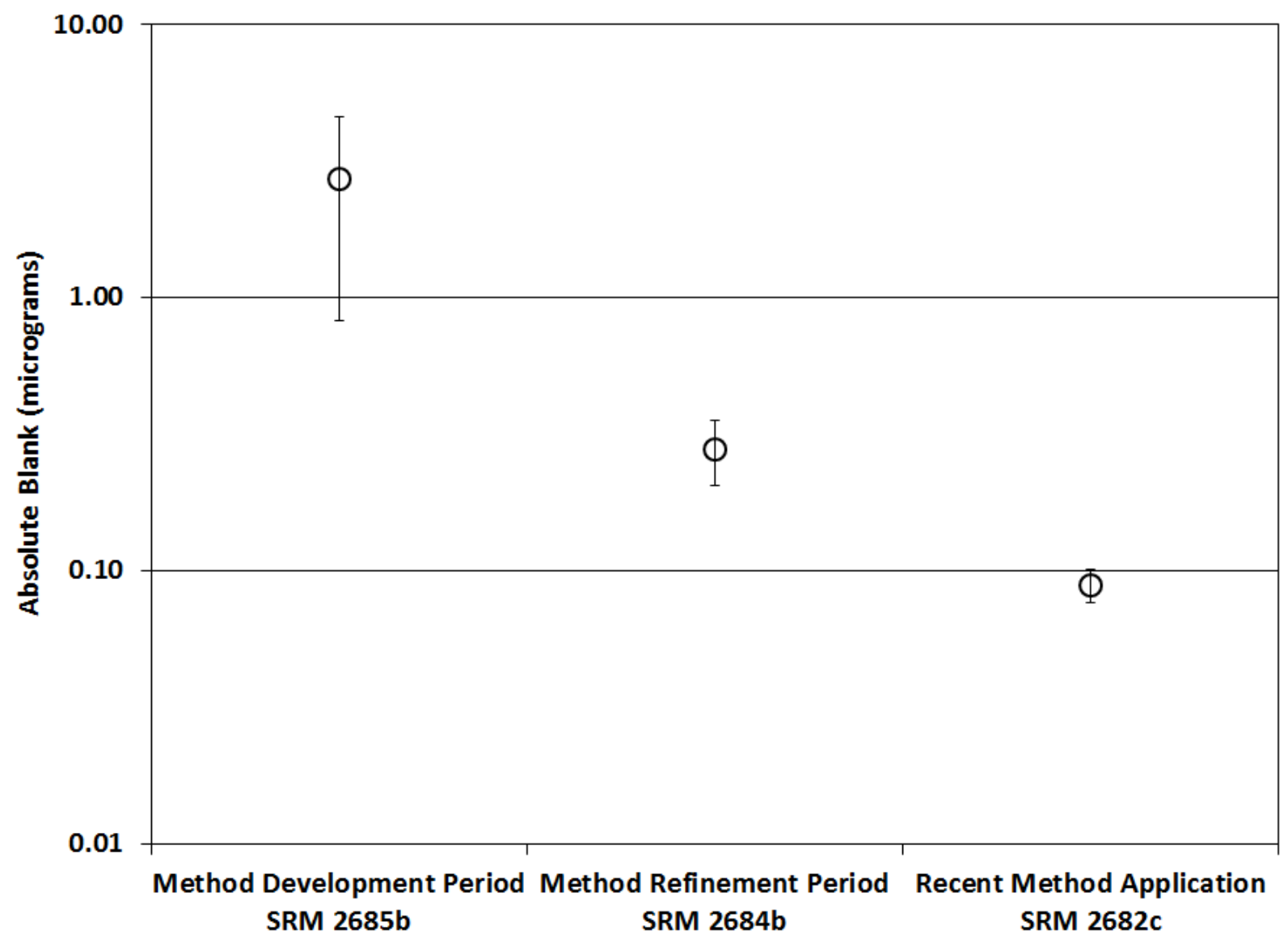


Supplemental Figure 2. Truncated Kragten spreadsheet for SRM 2682c (10 of 34 input columns shown). The most significant sources of uncertainty are readily apparent in the last data column (uncertainties calculated relative to the total variance). The results are based on a single permutation of the ID-MIC-SF-ICPMS measurement function ( 1 of 32 possible permutations, based on individual sample and spike solution pairs). The S mass fraction data for SRM $2682 \mathrm{c}$ described in the text are based on the average of all permutations $(0.4902 \% \pm$ $0.0068 \%$ ).

\begin{tabular}{|c|c|c|c|c|c|c|c|c|c|c|c|c|}
\hline & & $\mathrm{u}(\mathrm{az})$ & $u(a x)$ & $\mathrm{u}(\mathrm{dx})$ & $u(m x)$ & $u\left(m^{\prime} y\right)$ & $u(R b)$ & $u\left(R^{\prime} b\right)$ & $\mathrm{u}(\mathrm{Rep})$ & $u(d t)$ & $\mathrm{u}(\mathrm{Bgd})$ & \\
\hline & & 0.019 & 0.019 & 0.000 & 0.000 & 0.000 & 0.002 & 0.002 & 0.005 & 0.001 & 0.002 & \\
\hline & $\boldsymbol{\nu}_{i}$ & 99999.000 & 99999.000 & 4.000 & 99999.000 & 99999.000 & 4.000 & 4.000 & 7.000 & 99999.000 & 6.000 & \\
\hline Quantity Name, Symbol & $\begin{array}{l}\text { Quantity } \\
\text { Value }\end{array}$ & \multicolumn{10}{|c|}{ Quantity Value $+u_{i}$} & $\operatorname{rel}\left(c_{i} u_{i}\right)^{2}$ \\
\hline$a z$ & 32.064 & 32.083 & 32.064 & 32.064 & 32.064 & 32.064 & 32.064 & 32.064 & 32.064 & 32.064 & 32.064 & $0.00 \%$ \\
\hline$a x$ & 32.063 & 32.063 & 32.082 & 32.063 & 32.063 & 32.063 & 32.063 & 32.063 & 32.063 & 32.063 & 32.063 & $0.76 \%$ \\
\hline $\mathrm{dx}$ & 0.882 & 0.882 & 0.882 & 0.882 & 0.882 & 0.882 & 0.882 & 0.882 & 0.882 & 0.882 & 0.882 & $0.25 \%$ \\
\hline $\mathrm{mx}$ & 0.105 & 0.105 & 0.105 & 0.105 & 0.105 & 0.105 & 0.105 & 0.105 & 0.105 & 0.105 & 0.105 & $0.03 \%$ \\
\hline m'y & 0.900 & 0.900 & 0.900 & 0.900 & 0.900 & 0.900 & 0.900 & 0.900 & 0.900 & 0.900 & 0.900 & $0.00 \%$ \\
\hline $\mathrm{Rb}$ & 0.563 & 0.563 & 0.563 & 0.563 & 0.563 & 0.563 & 0.564 & 0.563 & 0.563 & 0.563 & 0.563 & $19.62 \%$ \\
\hline$R^{\prime} b$ & 0.666 & 0.666 & 0.666 & 0.666 & 0.666 & 0.666 & 0.666 & 0.668 & 0.666 & 0.666 & 0.666 & $19.23 \%$ \\
\hline Ry1 & 842.908 & 842.908 & 842.908 & 842.908 & 842.908 & 842.908 & 842.908 & 842.908 & 842.908 & 842.908 & 842.908 & $0.00 \%$ \\
\hline my & 0.072 & 0.072 & 0.072 & 0.072 & 0.072 & 0.072 & 0.072 & 0.072 & 0.072 & 0.072 & 0.072 & $0.06 \%$ \\
\hline $\mathrm{mz}$ & 0.909 & 0.909 & 0.909 & 0.909 & 0.909 & 0.909 & 0.909 & 0.909 & 0.909 & 0.909 & 0.909 & $0.00 \%$ \\
\hline $\mathrm{cz}$ & 158.288 & 158.288 & 158.288 & 158.288 & 158.288 & 158.288 & 158.288 & 158.288 & 158.288 & 158.288 & 158.288 & $1.22 \%$ \\
\hline cblk & 0.028 & 0.028 & 0.028 & 0.028 & 0.028 & 0.028 & 0.028 & 0.028 & 0.028 & 0.028 & 0.028 & $0.00 \%$ \\
\hline $\mathrm{Kb}$ & 0.915 & 0.915 & 0.915 & 0.915 & 0.915 & 0.915 & 0.915 & 0.915 & 0.915 & 0.915 & 0.915 & $0.91 \%$ \\
\hline k'b & 0.915 & 0.915 & 0.915 & 0.915 & 0.915 & 0.915 & 0.915 & 0.915 & 0.915 & 0.915 & 0.915 & $0.89 \%$ \\
\hline Ky1 & 1.000 & 1.000 & 1.000 & 1.000 & 1.000 & 1.000 & 1.000 & 1.000 & 1.000 & 1.000 & 1.000 & $0.00 \%$ \\
\hline $\mathrm{R} \times 1$ & 0.044 & 0.044 & 0.044 & 0.044 & 0.044 & 0.044 & 0.044 & 0.044 & 0.044 & 0.044 & 0.044 & $0.09 \%$ \\
\hline $\mathrm{R} \times 2$ & 0.008 & 0.008 & 0.008 & 0.008 & 0.008 & 0.008 & 0.008 & 0.008 & 0.008 & 0.008 & 0.008 & $0.00 \%$ \\
\hline $\mathrm{R} \times 3$ & 1.000 & 1.000 & 1.000 & 1.000 & 1.000 & 1.000 & 1.000 & 1.000 & 1.000 & 1.000 & 1.000 & $0.04 \%$ \\
\hline $\mathrm{R} \times 4$ & 0.000 & 0.000 & 0.000 & 0.000 & 0.000 & 0.000 & 0.000 & 0.000 & 0.000 & 0.000 & 0.000 & $0.00 \%$ \\
\hline Rz1 & 0.044 & 0.044 & 0.044 & 0.044 & 0.044 & 0.044 & 0.044 & 0.044 & 0.044 & 0.044 & 0.044 & $0.07 \%$ \\
\hline $\mathrm{Rz} 2$ & 0.008 & 0.008 & 0.008 & 0.008 & 0.008 & 0.008 & 0.008 & 0.008 & 0.008 & 0.008 & 0.008 & $0.00 \%$ \\
\hline Rz3 & 1.000 & 1.000 & 1.000 & 1.000 & 1.000 & 1.000 & 1.000 & 1.000 & 1.000 & 1.000 & 1.000 & $0.04 \%$ \\
\hline Rz4 & 0.000 & 0.000 & 0.000 & 0.000 & 0.000 & 0.000 & 0.000 & 0.000 & 0.000 & 0.000 & 0.000 & $0.00 \%$ \\
\hline $\mathrm{K} \times 1$ & 1.000 & 1.000 & 1.000 & 1.000 & 1.000 & 1.000 & 1.000 & 1.000 & 1.000 & 1.000 & 1.000 & $0.01 \%$ \\
\hline $\mathrm{K} \times 2$ & 1.000 & 1.000 & 1.000 & 1.000 & 1.000 & 1.000 & 1.000 & 1.000 & 1.000 & 1.000 & 1.000 & $0.00 \%$ \\
\hline $\mathrm{K} \times 3$ & 1.000 & 1.000 & 1.000 & 1.000 & 1.000 & 1.000 & 1.000 & 1.000 & 1.000 & 1.000 & 1.000 & $0.01 \%$ \\
\hline $\mathrm{Kx} 4$ & 1.000 & 1.000 & 1.000 & 1.000 & 1.000 & 1.000 & 1.000 & 1.000 & 1.000 & 1.000 & 1.000 & $0.00 \%$ \\
\hline Kz1 & 1.000 & 1.000 & 1.000 & 1.000 & 1.000 & 1.000 & 1.000 & 1.000 & 1.000 & 1.000 & 1.000 & $0.01 \%$ \\
\hline $\mathrm{Kz} 2$ & 1.000 & 1.000 & 1.000 & 1.000 & 1.000 & 1.000 & 1.000 & 1.000 & 1.000 & 1.000 & 1.000 & $0.00 \%$ \\
\hline $\mathrm{Kz} 3$ & 1.000 & 1.000 & 1.000 & 1.000 & 1.000 & 1.000 & 1.000 & 1.000 & 1.000 & 1.000 & 1.000 & $0.01 \%$ \\
\hline Kz4 & 1.000 & 1.000 & 1.000 & 1.000 & 1.000 & 1.000 & 1.000 & 1.000 & 1.000 & 1.000 & 1.000 & $0.00 \%$ \\
\hline Rep & 1.000 & 1.000 & 1.000 & 1.000 & 1.000 & 1.000 & 1.000 & 1.000 & 1.005 & 1.000 & 1.000 & $49.93 \%$ \\
\hline $\mathrm{dt}$ & 1.000 & 1.000 & 1.000 & 1.000 & 1.000 & 1.000 & 1.000 & 1.000 & 1.000 & 1.001 & 1.000 & $1.10 \%$ \\
\hline \multirow{3}{*}{ MF Value, $Y$} & 1.000 & 1.000 & 1.000 & 1.000 & 1.000 & 1.000 & 1.000 & 1.000 & 1.000 & 1.000 & 1.002 & $5.72 \%$ \\
\hline & 4785.343 & 4785.343 & 4788.107 & 4783.772 & 4784.816 & 4785.282 & 4771.343 & 4799.202 & 4807.676 & 4788.659 & 4792.900 & $100.00 \%$ \\
\hline & & -0.001 & 2.763 & -1.571 & -0.528 & -0.061 & -14.000 & 13.859 & 22.333 & 3.315 & 7.557 & $c_{i} u_{i}$ \\
\hline Standard Uncertainty, $\boldsymbol{u}_{\mathrm{c}}$ & 31.605 & 0.000 & 7.636 & 2.468 & 0.278 & 0.004 & 196.011 & 192.065 & 498.751 & 10.992 & 57.104 & $\left(c_{i} u_{i}\right)^{2}$ \\
\hline Degrees of Freedom, $v$ & 18.172 & $0.00 \%$ & $0.76 \%$ & $0.25 \%$ & $0.03 \%$ & $0.00 \%$ & $19.62 \%$ & $19.23 \%$ & $49.93 \%$ & $1.10 \%$ & $5.72 \%$ & $\operatorname{rel}\left(c_{j} u_{j}\right)^{2}$ \\
\hline Coverage Factor, $k$ & 2.101 & -0.031 & 149.279 & -5423.971 & -45683.649 & $\begin{aligned}-5319.927 \\
\end{aligned}$ & -9270.188 & 7750.851 & 4785.343 & 4785.343 & 4785.343 & $c_{i}$ \\
\hline Expanded Uncertainty, $U$ & 66.400 & & & & & & & & & & 0.014 & $U_{t}$ \\
\hline \multicolumn{13}{|l|}{$\leftarrow$ Show/Hide Extra Information } \\
\hline \multirow{3}{*}{ MF Value, $Y$} & \multicolumn{11}{|c|}{ Quantity Value - $u_{i}$ (Results Only) } & \\
\hline & 4785.343 & 4785.344 & 4782.580 & 4786.915 & 4785.871 & 4785.405 & 4799.426 & 4771.484 & 4763.010 & 4782.028 & 4777.786 & \\
\hline & & 0.001 & -2.763 & 1.572 & 0.528 & 0.061 & 14.083 & -13.859 & -22.333 & -3.315 & -7.557 & $c_{i} u_{i}$ \\
\hline Standard Uncertainty, $u_{\mathrm{c}}$ & 31.642 & 0.000 & 7.636 & 2.471 & 0.278 & 0.004 & 198.323 & 192.064 & 498.751 & 10.992 & 57.104 & $\left(c_{i} u_{i}\right)^{2}$ \\
\hline Degrees of Freedom, $v$ & 18.181 & $0.00 \%$ & $0.76 \%$ & $0.25 \%$ & $0.03 \%$ & $0.00 \%$ & $19.81 \%$ & $19.18 \%$ & $49.81 \%$ & $1.10 \%$ & $5.70 \%$ & $\operatorname{rel}\left(c_{j} u_{j}\right)^{2}$ \\
\hline Coverage Factor, $k$ & 2.101 & 0.031 & -149.279 & 5427.535 & 45693.721 & 5320.064 & 9324.708 & -7750.821 & -4785.343 & -4785.343 & -4785.343 & $c_{i}$ \\
\hline Expanded Uncertainty, $U$ & 66.478 & & & & & & & & & & 0.014 & $u_{r}$ \\
\hline \multirow{2}{*}{\multicolumn{13}{|c|}{ — Show/Hide Extra Information }} \\
\hline & & & & & & & & & & & & \\
\hline \multicolumn{13}{|c|}{ Final Measurement Result } \\
\hline Measurement Function Value & $\begin{array}{c}\text { Standard } \\
\text { Uncertainty }\end{array}$ & $\begin{array}{l}\text { Degrees of } \\
\text { Freedom }\end{array}$ & $\begin{array}{c}\text { Coverage } \\
\text { Factor }\end{array}$ & $\begin{array}{l}\text { Expanded } \\
\text { Uncertainty }\end{array}$ & \multicolumn{2}{|c|}{$\begin{array}{c}\text { Relative Expanded } \\
\text { Uncertainty }\end{array}$} & $\begin{array}{c}\text { Unit } \\
\text { Conversion }\end{array}$ & $\begin{array}{c}\text { S Mass } \\
\text { Fraction } \\
\text { Value }\end{array}$ & $\begin{array}{c}\text { Expanded } \\
\text { Uncertainty } \\
U\end{array}$ & & & \\
\hline$Y$ & $u_{\mathrm{c}}$ & $v$ & $k$ & $u$ & \multicolumn{2}{|c|}{$U_{\mathrm{r}}$} & $\mathrm{mg} / \mathrm{kg}$ to $\%$ & $\%$ & $\%$ & & & \\
\hline 4785.343 & 31.624 & 18.167 & 2.101 & 66.439 & \multicolumn{2}{|c|}{0.014} & 10000 & 0.4785 & 0.0066 & & & \\
\hline \multirow{3}{*}{\multicolumn{2}{|c|}{ No Warning for Zero Sensitivity Coefficients }} & $u(\mathrm{az})$ & $u(\mathrm{ax})$ & $u(d x)$ & $u(m x)$ & $u\left(m^{\prime} y\right)$ & $u(R b)$ & $u\left(R^{\prime} b\right)$ & $u(R e p)$ & $u(T d t)$ & $u(B g d)$ & \\
\hline & & $0.00 \%$ & $0.76 \%$ & $0.25 \%$ & $0.03 \%$ & $0.00 \%$ & $19.72 \%$ & $19.21 \%$ & $49.87 \%$ & $1.10 \%$ & $5.71 \%$ & $\operatorname{rel}\left(c_{j} u_{j}\right)^{2}$ \\
\hline & & -0.031 & 149.279 & -5425.753 & -45688.685 & -5319.995 & -9297.448 & 7750.836 & 4785.343 & 4785.343 & 4785.343 & $c_{i}$ \\
\hline
\end{tabular}

